\title{
Imaging Improves Diagnosis of Dementia with Lewy Bodies
}

\author{
Masaru Tateno \\ Seiju Kobayashi \\ Toshikazu Saito \\ Department of Neuropsychiatry, \\ Sapporo Medical University \\ School of Medicine, Sapporo, Japan
}

\section{Correspondence \\ Masaru Tateno, MD, PhD \\ Department of Neuropsychiatry, \\ Sapporo Medical University \\ School of Medicine, \\ South-1, West-16, Chuo-ku, \\ Sapporo 0608543, Japan \\ Tel +81-11-611-2111 (ext.3518) \\ Fax +81-11-644-3041 \\ E-mail tatema@sapmed.ac.jp}

(cc) This is an Open Access article distributed under the terms of the Creative Commons Attribution Non-Commercial License (http://creativecommons.org/licenses/by-nc/3.0) which permits unrestricted non-commercial use, distribution, and reproduction in any medium, provided the original work is properly cited.
Dementia with Lewy bodies (DLB) is the second most common cause of degenerative dementia after Alzheimer's disease (AD), and is clinically characterized by the progressive cognitive decline with fluctuations in cognition and alertness, recurrent visual hallucinations and Parkinsonism. Once these characteristic symptoms of DLB emerge, discriminating it from $\mathrm{AD}$ is relatively easy. However, in the early disease stages, the clinical symptoms of various types of dementias largely overlap and it is difficult to distinguish DLB from other neurodegenerative dementias based on clinical manifestations alone. To increase the accuracy of antemortem diagnosis of DLB, the latest diagnostic criteria incorporate findings from 123I-metaiodobenzylguanidine (MIBG) myocardial scintigraphy, or from neuroimaging such as computed tomography (CT), magnetic resonance imaging (MRI), single photon emission computed tomography (SPECT), and positron emission tomography (PET). In the present guidelines, decreased dopamine transporter uptake revealed by SPECT or PET receives the greatest importance among various neuroimaging findings and is listed as one of the suggestive features. Supportive features that commonly present but are not proven to have diagnostic specificity include relatively-preserved medial-temporal-lobe structures, occipital hypoperfusion, and abnormal MIBG myocardial scintigraphy. In this paper, we review the major findings on various neuroimaging modalities and discuss the clinical usefulness of them for the diagnosis of DLB. Although there is not enough evidence to reach the conclusion, considering the accessibility in clinical practice, in our personal views, we recommend the use of brain-perfusion SPECT and MIBG myocardial scintigraphy to improve the diagnosis of DLB.

Psychiatry Invest 2009;6:233-240

Key Words Dementia, Dementia with Lewy bodies, Magnetic resonance imaging, Single photon emission computed tomography, Positron emission tomography, 123I-metaiodobenzylguanidine myocardial scintigraphy.

Received: September 27, 2009 Accepted: November 12, 2009 Available online: November 21, 2009

\section{Historical Background of Dementia with Lewy Bodies}

The concept of dementia with Lewy bodies (DLB) was first proposed in a case report by a Japanese psychiatrist, Dr. Kenji Kosaka, in 1976. ${ }^{1}$ Two years later, based on their neuropathological findings from three autopsied cases, Kosaka ${ }^{2}$ reported the characteristic distribution of Lewy bodies in cerebral cortices in the subjects with the later so-called "diffuse Lewy body disease (DLBD)". After the accumulation of similar cases, the term "Lewy body disease" was introduced and used to represent a spectrum of diseases involving an array of cognitive dysfunctions and motor symptoms. ${ }^{3}$ The spectrum could be divided into three types of disease according to the distributional pattern of Lewy bodies: the diffuse type, the transitional type and the brain stem and diencephalon type. ${ }^{4}$ The brain stem type is equivalent to Parkinson disease (PD), and the diffuse type is later designated as DLBD. Furthermore, Kosaka's group reviewed all reported DLBD cases in Japan and classified them into two subtypes; the common form contained various degrees of Alzheimer's pathology while the pure form never had such neuropathological findings. Then, in 1984, the 
term DLBD was proposed as a broader clinical entity which included various types of medical conditions with Lewy bodies. ${ }^{4}$ In short, Lewy body disease was referred to by various names such as Lewy Body disease, Lewy Body dementia, diffuse Lewy Body disease and DLB. The mixture of these words confused the clinicians till they reached a certain degree of consensus. Based on the accumulating cases, the definition of the term "DLB" was proposed and approved at the first international workshop in $1995 .^{5}$ The publication of clinical guidelines for DLB highlighted a previously under-diagnosed condition and illuminated this clinical entity. ${ }^{6}$ However, several clinical studies revealed that the initial diagnostic criteria had insufficient sensitivity for reliable DLB recognition. ${ }^{7}$ To achieve acceptably-high sensitivity and specificity, the guidelines were revised and released anew in $2005 .^{8}$ The revised version of guidelines has been well accepted and used worldwide both for clinical and research purposes.

\section{Neuroimaging Studies in Dementia with Lewy Bodies}

Currently, DLB is reported to be the second most common cause of degenerative dementia after Alzheimer's disease (AD). ${ }^{7-11}$ Since DLB causes characteristic impairments and disabilities such as neuroleptic hypersensitivity which may increase mortality, its prompt and correct diagnosis is very important. However, because the clinical symptoms of DLB and AD overlap in the early disease stages, it is difficult to distinguish DLB from AD based on clinical manifestations alone.

To increase the accuracy of antemortem diagnosis of DLB, the latest diagnostic criteria (Table 1) incorporate findings from 123I-metaiodobenzylguanidine (MIBG) myocardial scintigraphy, or from neuroimaging such as computed tomography (CT), magnetic resonance imaging (MRI), single photon emission computed tomography (SPECT), and positron emission tomography (PET). ${ }^{8}$ This imaging provides highly objective and precise data that contributes to the clinical diagnosis of DLB. For example, among three suggestive features in the diagnostic criteria of DLB: rapid eye movement (REM) sleep behavior disorder, severe neuroleptic sensitivity and low dopamine transporter uptake, the measurement of dopamine transporter activity in striatum by SPECT or PET enables the most objective and highly accurate assessment of characteristic abnormalities in DLB and could be a helpful diagnostic aid in discriminating DLB and AD. Supportive

TABLE 1. The latest guidelines for the diagnosis of DLB

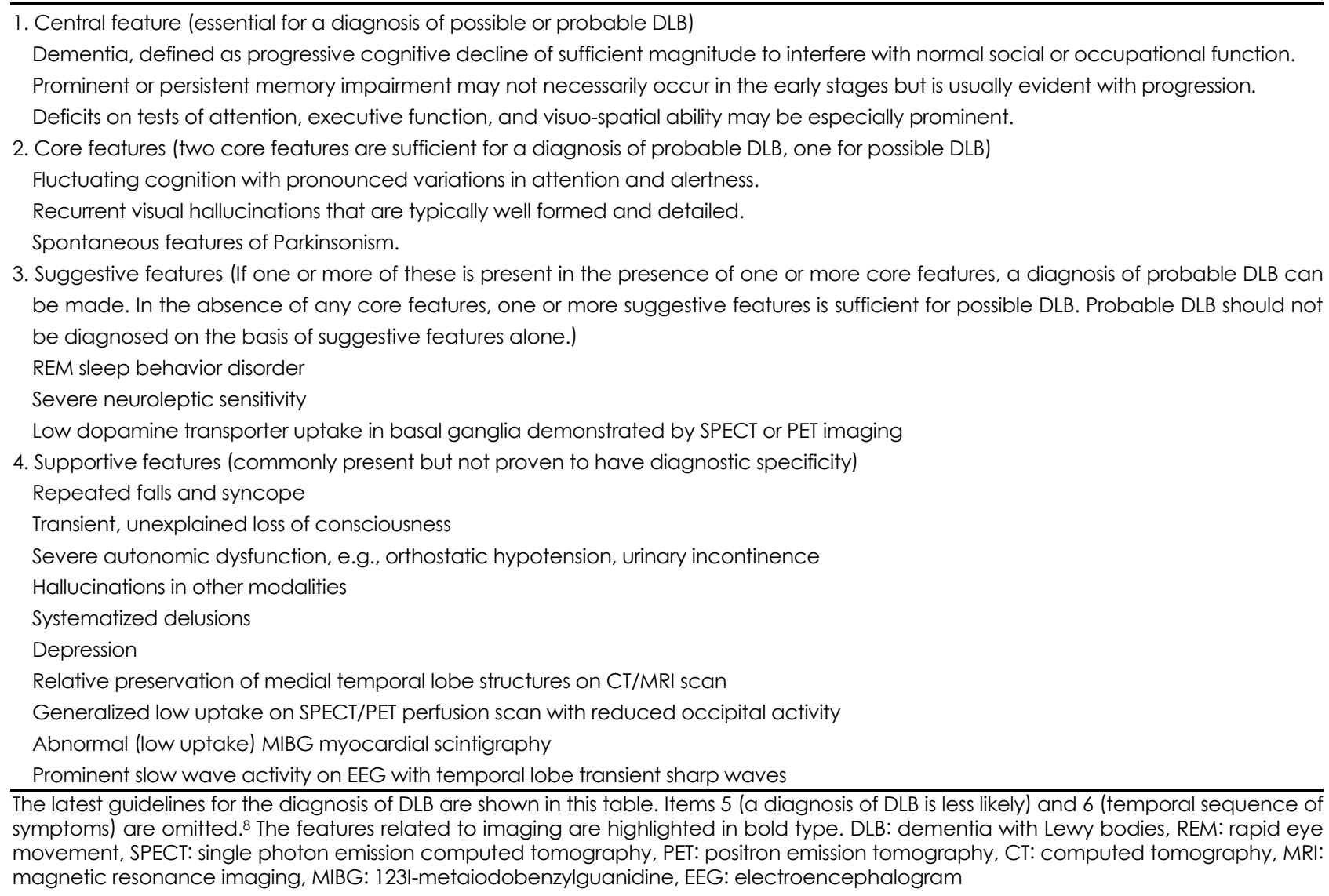


features include relatively preserved medial temporal lobe structures, occipital hypoperfusion/metabolism, and decreased MIBG uptake on myocardial scintigraphy. The results of CT, MRI, PET and brain-perfusion SPECT could be evaluated by statistical analyzing programs objectively.

In this paper, we give a brief overview of studies in DLB using various imaging modalities.

\section{Morphological neuroimaging studies}

Morphological neuroimaging, such as brain CT and MRI, is often performed for the clinical diagnosis of various types of dementia. ${ }^{12}$ The main aims of this imaging are the detection of cerebrovascular diseases, spaceoccupying legions such as hematoma and brain tumor, and the evaluation of the degree of cerebral atrophy. ${ }^{13-15}$ In regard to the diagnosis of DLB, there is no confirmed characteristics with a significant diagnostic value from morphological neuroimaging. ${ }^{12}$ In the consensus guidelines, ${ }^{8}$ relatively-preserved medial-temporal-lobe structures on CT/MRI are alone listed as one of the supportive features in DLB. These are commonly present but are not proven to have diagnostic specificity.

\section{Region-of-Interest Based Analyses}

Previous morphological neuroimaging studies used region-of-interest (ROI) based analyses to assess the degree of cerebral atrophy. In this method, one needed to place the ROIs manually, and only selected regions could be investigated.

Morphological neuroimaging studies using visual inspection or ROI techniques demonstrated that the extent of medial temporal lobe atrophy was severer in $\mathrm{AD}$ compared to DLB. ${ }^{16-20}$ Hashimoto et al. ${ }^{17}$ reported that hippocampal volume in DLB was significantly larger than that in $\mathrm{AD}$, but significantly smaller than in the normal control. In their study, no significant differences were found in the amygdala and whole-brain volume between DLB and AD, though the amygdala and whole brain were smaller in DLB compared to the control. These findings were consistent with the results of the study by Barber et al. ${ }^{16}$ that demonstrated that the subjects with DLB had significantly larger temporal-lobe, hippocampal, and amygdala volumes than those with $\mathrm{AD}$. Their results also reported that significant ventricular dilation was observed in the subjects with $\mathrm{AD}$, vascular dementia $(\mathrm{VaD})$ and DLB, and only the subjects with DLB had a relatively preserved volume of the cerebrum. The medial temporal lobe atrophy in DLB has been reported to be related to age and cognitive impairment. ${ }^{19,20}$ Therefore, clinicians need to take the patients' history carefully and examine clinical symptoms thoroughly before interpreting the re- sults of neuroimaging.

Besides the temporal lobe, other cerebral structures have been studied morphologically. Almeida et al. ${ }^{21}$ reported that there were no significant differences in caudate nucleus volume in DLB, AD, PD, PD with dementia (PDD) and controls. However, Cousins's group demonstrated that the volume of putamen was smaller in DLB compared to $\mathrm{AD}$ and normal controls. ${ }^{22}$ Regarding the atrophy of substantia innominata, Hanyu and his colleagues ${ }^{23}$ showed significant reduction of the volume only in DLB, while the volume was preserved in $\mathrm{AD}, \mathrm{VaD}$ and the control.

\section{Voxel-Based Morphometry}

Voxel-based morphometry (VBM) is one of the neuroimaging analysis techniques that investigate morphological differences by using statistical parametric mapping (SPM). ${ }^{24,25}$ VBM enables objective and quantitative analysis of brain structure.

Compared to the normal control, decreased volume of the temporal and frontal lobes and insular cortex was found in DLB by Burton's group. ${ }^{26}$ The MRI study done by Whitwell et al. ${ }^{27}$ revealed decreased volume of the dorsal midbrain, substantia innominata and hypothalamus. Brenneis et al. ${ }^{28}$ demonstrated the atrophy of lateral prefrontal cortex and left premotor cortex in DLB.

In regard to the comparison between DLB and $\mathrm{AD}, \mathrm{AD}$ patients showed decreased volume in the medial temporal lobe, hippocampus, amygdala and thalamus in Burton's study, ${ }^{26}$ in the hippocampus and temporo-parietal cortex in Whitwell's study, ${ }^{27}$ and in the temporal and frontal lobe in Beyer's study. ${ }^{29}$

Taken together, we can conclude that the relativelypreserved volume of the medial temporal lobe could be a support for the diagnosis of DLB, especially for the discrimination of DLB from AD.

\section{Diffusion Tensor Magnetic Resonance Imaging}

Diffusion tensor imaging (DTI) is an advanced neuroimaging technique that performs a more sensitive investigation of tissue microstructure compared to conventional imaging methods, and enables the extraction of white matter connections of the brain using tractography. ${ }^{30-33}$ A number of studies have reported the usefulness of DTI for investigating the pathophysiological mechanisms of DLB. ${ }^{34,35}$ Firbank et al. ${ }^{36,37}$ demonstrated a decreased fractional anisotropy in the parietal lobe in DLB and an increased apparent diffusion coefficient in the left temporal lobe in $\mathrm{AD}$, suggesting the region-specific disruption of the connectivity in respective dementias. Bozzali et al. ${ }^{38}$ revealed abnormalities in brain regions with long connecting tracts, which suggested the involvement of association cortices in neurodegenerative 
process in DLB. This group also demonstrated that no significant abnormality was found in the occipital region of AD subjects and normal controls, and suggested that DTI could be a support tool for the diagnosis of DLB. ${ }^{39}$ Ota and his colleagues ${ }^{40}$ applied DTI to examine the mechanisms of visual hallucination in DLB and indicated the possible involvement of inferior longitudinal fasciculus which played an important role in visuo-spatial cognition.

\section{Functional neuroimaging studies}

The initial consensus guidelines for the clinical diagnosis of $\mathrm{DLB}^{5}$ proved to be insufficiently sensitive for reliable DLB recognition. ${ }^{7}$ To increase the accuracy of the clinical diagnosis of DLB, the latest diagnostic criteria incorporate findings from SPECT or PET, and include reduced occipital perfusion as a supportive feature. ${ }^{8}$

\section{Single Photon Emission Computed Tomography}

Brain-perfusion SPECT is often performed for the diagnosis of dementia, along with CT and/or MRI. Characteristic hypoperfusion in the hippocampus, posterior cingulate gyrus and temporoparietal cortex is very useful for the early detection of AD. ${ }^{41-44}$ The most important finding on brain-perfusion SPECT in DLB is occipital hypoperfusion. This hypoperfusion is listed as a supportive feature in the consensus guidelines. ${ }^{8}$

To increase the accuracy of the clinical evaluation and to achieve the highest possible agreement between different readers, algorithms for analyzing SPECT images have been developed and used in the clinical setting. Two widely used methods are SPM ${ }^{45,46}$ and three-dimensional stereotactic surface projection (3D-SSP). ${ }^{47-51}$ In both algorithms, SPECT images are anatomically standardized and statistically analyzed. Both SPM and 3D-SSP enable us to detect cerebral regions with lowered blood perfusion with statistical significance. Since SPM uses the ttest for statistical analysis, when the degrees of freedom of the sample are small, the sensitivity can be lowered while specificity remains high. 3D-SSP has high sensitivity and specificity. However, as the images are projected to the surface of the schematic brain, it is difficult to determine the exact region of abnormal blood flow. To make better use of the advantages discussed above, Matsuda et al. developed a new qualitative analysis method named eZIS. ${ }^{52-55}$ This method is accompanied by a database prepared from age-matched normal controls, and compares the results of individual subjects with the database for statistical analysis. The extent of the decrease of cerebral blood flow is expressed as a set of $\mathrm{Z}$-values. Each value equals the division of the difference between the mean of the normal controls and the result from the subject by the standard deviation of the normal controls. The Z-value results are shown as a projection chart on a schematic brain. Furthermore, Takeuchi et al. developed a fully-automated rCBF quantification method, 3DSRT, which allowed objective assessment of rCBF by setting the ROIs identically on anatomically-standardized SPECT images. ${ }^{56-59}$ 3DSRT includes anatomical standardization of images employing SPM which is used only for that purpose, rCBF quantification using a three-dimensional stereotactic ROI template, calculation of $\mathrm{CBF}$ and display of the results.

The initial SPECT studies using 99mTc-hexamethyl propyleneamine oxime (HMPAO) as a tracer suggested limited value of such studies for the differential diagnosis of DLB from AD. ${ }^{60-62}$ However, the following research demonstrated that occipital hypoperfusion revealed by brain-perfusion SPECT had high enough sensitivity and specificity to distinguish DLB from other dementias.

Ishii et al. ${ }^{63}$ performed HMPAO-SPECT in DLB, AD and healthy control, and analyzed their results by using SPM. The analysis results revealed decreased cerebral blood flow in the occipital lobes. Lobotesis et al. ${ }^{64}$ reported that blood perfusion in AD and DLB differed only in occipital areas and distinguished DLB from AD and control subjects with a $65 \%$ sensitivity and $87 \%$ specificity which were equal to Varma's study. ${ }^{65}$ Colloby and his colleagues ${ }^{66}$ investigated the results of the HMPAOSPECT performed in 48 AD, 23 DLB and 20 control subjects, and indicated hypoperfusion in both parietal and occipital regions of the brain in DLB compared to the AD group.

Pasquier et al. ${ }^{67}$ used $99 \mathrm{mTc}$-ethylcysteinate dimer (ECD) as a tracer for their brain-perfusion-SPECT study and obtained a similar sensitivity of $65 \%$ for the diagnosis of DLB. Our group performed ECD-SPECT in 25 DLB subjects and analyzed the results by using eZIS. ${ }^{68}$ The sensitivity calculated in our study was $68 \%$ and this value was equivalent to the previous studies. On the whole, the sensitivity of brain-perfusion SPECT has been reported to be around $60-70 \%$.

We performed quantitative analysis of brain-perfusion SPECT in DLB and demonstrated the clinical usefulness of an automated cerebral blood flow quantification program, 3DSRT, for the diagnosis of DLB. ${ }^{69}$ 3DSRT enabled us to estimate the blood perfusion in the region of interest. In the comparison of the usefulness of different SPECT analyzing programs, the 3DSRT analysis of brainperfusion SPECT in DLB showed higher sensitivity (76\%) compared to that of eZIS $(68 \%){ }^{68}$

\section{Positron Emission Tomography}

Cerebral metabolism is often measured for the clini- 
cal diagnosis of dementias by using fluorodeoxyglucose (FDG) and PET $^{70}$ Characteristic regional hypometabolism could be useful to differentiate DLB from other dementias.

Albin and his colleagues ${ }^{71}$ were the first to report hypometabolism in the occipital association cortex and primary visual cortex in DLB. In the following year, Imamura and his colleagues ${ }^{72}$ verified Albin's findings by investigating a bigger sample. Ishii et al. ${ }^{73}$ performed FDG-PET and investigated the regional cerebral metabolic rate of glucose in three groups: DLB, AD and controls. Their results demonstrated that the decreased metabolic rate in the occipital region could distinguish DLB from $\mathrm{AD}$ with a sensitivity and specificity of $92 \%$ each. His group suggested that occipital hypometabolism might be associated with visual hallucinations in DLB. ${ }^{74}$ The PET study conducted by Higuchi's group indicated that the glucose metabolism was significantly reduced in the visual association cortex of DLB subjects compared to the $\mathrm{AD}$ group. ${ }^{75}$ Minoshima et al. ${ }^{76}$ examined cerebral glucose metabolism in the subjects with autopsy-confirmed $\operatorname{DLB}(\mathrm{n}=11)$ and $\mathrm{AD}(\mathrm{n}=10)$ along with 53 probable $\mathrm{AD}$ subjects including 13 cases who later received a clinical diagnosis of DLB. In their study, hypometabolism in the primary visual cortex distinguished DLB from AD with $90 \%$ sensitivity and $80 \%$ specificity.

\section{Dopamine Transporter Imaging}

In the latest criteria for the clinical diagnosis of DLB, decreased dopamine transporter (DAT) uptake in the basal ganglia is listed as one of the suggestive features. ${ }^{8}$ If one or more suggestive features is present in combination with one or more core features, a diagnosis of probable DLB can be made, whereas in the absence of any core features, one or more suggestive features is defined as possible DLB. Thus, DAT imaging receives the highest priority among various neuroimagings. To investigate the striatal DAT function in DLB, two kinds of ligands for SPECT imaging are used: [123I]-2beta-carbometoxy3beta-(4-iodophenyl)-N-(3-fluoropropyl) nortropane (FPCIT) and [123I]-2beta-carbomethoxy-3beta-(4-iodophenyl)tropane (beta-CIT). Donnemiller et al. ${ }^{62}$ performed beta-CIT SPECT on 7 probable DLB, 6 probable AD and 3 normal controls, and indicated that low DAT function was useful to discriminate DLB from AD and normal controls. His group also performed SPECT imaging by using beta-CIT on 20 DLB patients, 24 subjects with PD and 10 normal controls, and demonstrated that DAT imaging might be useful to distinguish DLB and $\mathrm{PD} .{ }^{77}$ As to FP-CIT, O'Brien's group used this ligand and performed DAT imaging on probable/possible DLB, PD, PDD, AD and normal controls. ${ }^{78}$ Their results demonst- rated that the sensitivity, specificity and positive predictive value for the differentiation of DLB from AD were $78 \%, 94 \%$ and $90 \%$, respectively, though it was difficult to separate DLB from PD with and without dementia. McKeith et al. lead a multicenter study about FP-CIT SPECT on DLB and assessed 326 patients including probable $(n=94)$ or possible $(n=57)$ DLB or 147 non-DLB dementia. ${ }^{79}$ They calculated a mean sensitivity of $77.7 \%$ for discriminating probable DLB and specificity of $90.4 \%$ for excluding non-DLB dementia. Based on these findings, low DAT uptake was given the diagnostic importance as a suggestive feature of DLB.

Despite these clinical studies, the usefulness of DAT imaging for the diagnosis of DLB remains controversial. ${ }^{80}$ Further studies are awaited.

\section{I-Metaiodobenzylguanidine}

MIBG is a physiological analogue of norepinephrine and competes with it for neuronal uptake at the sympathetic nerve terminal. ${ }^{81-83}$ MIBG myocardial scintigraphy was originally used to assess myocardial sympathetic nerve damage in heart disease ${ }^{84-86}$ Later this method was applied to detect cardiac sympathetic denervation in PD and clinically used to discriminate PD from other neurological disorders with extrapyramidal signs (EPS)..$^{87-89}$ The heart-to-mediastinum ratio of myocardial MIBG uptake and the washout rate in percent are used to assess the severity of the postganglionic cardiac sympathetic nerve denervation. Recent studies have demonstrated that MIBG myocardial scintigraphy is useful for the clinical diagnosis of DLB..$^{90-93}$ The latest guidelines include abnormal MIBG myocardial scintigraphy as one of the supportive features which commonly present in DLB but are not proven to have diagnostic specificity.

Yoshita et al. ${ }^{90}$ first reported the clinical usefulness of MIBG scintigraphy to distinguish DLB from other dementias by investigating 14 subjects with DLB. The clinical difficulty of discriminating DLB from AD stems from the fact that there are many AD cases with EPS and DLB cases without Parkinsonism. MIBG myocardial scintigraphy detected disturbances of cardiac sympathetic nerves in patients with DLB regardless of clinically-evident Parkinsonism, whereas AD patients with EPS showed no significant decrease of MIBG uptake. ${ }^{94,95}$ Yoshita's group performed MIBG myocardial scintigraphy on 37 probable DLB patients including 7 cases without EPS, 42 probable AD subjects and 10 normal controls. ${ }^{95}$ They reported that setting the cut-off value of the heart-to-mediastinum ratio at 1.68 yielded $100 \%$ sensitivity and specificity for differentiating DLB from AD. Similarly, setting the washout-rate cut-off value at $23.6 \%$ yielded $87 \%$ sensitivity and $83 \%$ specificity. 
In comparing the clinical value of brain-perfusion SPECT and MIBG myocardial scintigraphy, Hanyu et al. ${ }^{96}$ observed decreased heart-to-mediastinum ratios in all 19 DLB patients, while only 14 of them showed occipital hypoperfusion. They concluded that MIBG myocardial scintigraphy could improve sensitivity in the detection of DLB. The results of our study were consistent with those from Hanyu's study. Twenty-four of 25 subjects (96\%) had decreased cardiac MIBG uptake in the delayed image (3 hours after injection), while occipital hypoperfusion was observed in only $68 \%$ and $76 \%$ of them by applying, respectively, eZIS and 3DSRT. ${ }^{68}$ Most studies on MIBG myocardial scintigraphy thus far have been conducted by Japanese groups. However, a Spanish team recently reported that a heart-to-mediastinum-ratio cutoff value of 1.36 differentiated DLB from other dementias with a sensitivity of $94 \%$ and a specificity of $96 \%{ }^{97}$

At the 4th International Workshop on DLB and PDD, there was a heated debate about whether abnormal MIBG myocardial scintigraphy should be included as a suggestive feature for DLB. At present, there is not enough evidence to thoroughly validate the usefulness of MIBG myocardial scintigraphy in the diagnosis of DLB, and we still need to accumulate more data.

\section{Conclusion}

DLB has been reported to be the second most common type of degenerative dementia. However, DLB is often under-diagnosed and its discrimination from other dementias is very difficult, especially at its early stages. Since DLB has specific impairments and functional disabilities, its early and accurate diagnosis is very important for a better prognosis. To achieve such an improved prognosis, neuroimaging studies and MIBG myocardial scintigraphy play important roles. Further research is needed to establish their clinical usefulness even further.

\section{- Acknowledgments}

The neuroimaging studies by the authors' group were supported by a Grant-in-Aid for Young Scientists (B)(MT: 20790852) from the Japan Society for the Promotion of Science (JSPS).

\section{REFERENCES}

1. Kosaka K, Oyanagi S, Matsushita M, Hori A. Presenile dementia with Alzheimer-, Pick- and Lewy-body changes. Acta Neuropathol 1976; 36:221-233.

2. Kosaka K. Lewy bodies in cerebral cortex, report of three cases. Acta Neuropathol 1978;42:127-134.

3. Rosenblum WI, Ghatak NR. Lewy bodies in the presence of Alzheimer's disease. Arch Neurol 1979;36:170-171.

4. Kosaka K, Yoshimura M, Ikeda K, Budka H. Diffuse type of Lewy body disease: progressive dementia with abundant cortical Lewy bodies and senile changes of varying degree--a new disease? Clin Neu- ropathol 1984;3:185-192.

5. McKeith IG, Galasko D, Kosaka K, Perry EK, Dickson DW, Hansen LA, et al. Consensus guidelines for the clinical and pathologic diagnosis of dementia with Lewy bodies (DLB): report of the consortium on DLB international workshop. Neurology 1996;47:1113-1124.

6. McKeith IG, Ballard CG, Perry RH, Ince PG, O'Brien JT, Neill D, et al. Prospective validation of consensus criteria for the diagnosis of dementia with Lewy bodies. Neurology 2000;54:1050-1058.

7. McKeith I, Mintzer J, Aarsland D, Burn D, Chiu H, Cohen-Mansfield J, et al. Dementia with Lewy bodies. Lancet Neurol 2004;3:19-28.

8. McKeith IG, Dickson DW, Lowe J, Emre M, O’Brien JT, Feldman H, et al. Diagnosis and management of dementia with Lewy bodies: third report of the DLB Consortium. Neurology 2005;65:1863-1872.

9. Gómez-Tortosa E, Ingraham AO, Irizarry MC, Hyman BT. Dementia with Lewy bodies. J Am Geriatr Soc 1998;46:1449-1458.

10. Weisman D, McKeith I. Dementia with Lewy bodies. Semin Neurol 2007;27:42-47.

11. Rahkonen T, Eloniemi-Sulkava U, Rissanen S, Vatanen A, Viramo P, Sulkava R. Dementia with Lewy bodies according to the consensus criteria in a general population aged 75 years or older. J Neurol Neurosurg Psychiatry 2003;74:720-724.

12. Kemp PM, Holmes C. Imaging in dementia with Lewy bodies: a review. Nucl Med Commun 2007;28:511-519.

13. Varma AR, Adams W, Lloyd JJ, Carson KJ, Snowden JS, Testa HJ, et al. Diagnostic patterns of regional atrophy on MRI and regional cerebral blood flow change on SPECT in young onset patients with Alzheimer's disease, frontotemporal dementia and vascular dementia. Acta Neurol Scand 2002;105:261-269.

14. O'Brien JT, Erkinjuntti T, Reisberg B, Roman G, Sawada T, Pantoni L, et al. Vascular cognitive impairment. Lancet Neurol 2003;2:89-98.

15. Barber R, Scheltens P, Gholkar A, Ballard C, McKeith I, Ince P, et al. White matter lesions on magnetic resonance imaging in dementia with Lewy bodies, Alzheimer's disease, vascular dementia, and normal aging. J Neurol Neurosurg Psychiatry 1999;67:66-72.

16. Barber R, Gholkar A, Scheltens P, Ballard C, McKeith IG, O’Brien JT Medial temporal lobe atrophy on MRI in dementia with Lewy bodies. Neurology 1999;52:1153-1158.

17. Hashimoto M, Kitagaki H, Imamura T, Hirono N, Shimomura T, Kazui $\mathrm{H}$, et al. Medial temporal and whole-brain atrophy in dementia with Lewy bodies: a volumetric MRI study. Neurology 1998;51:357-362.

18. Barber R, Ballard C, McKeith IG, Gholkar A, O’Brien JT. MRI volumetric study of dementia with Lewy bodies: a comparison with AD and vascular dementia. Neurology 2000;54:1304-1309.

19. Tam CW, Burton EJ, McKeith IG, Burn DJ, O’Brien JT. Temporal lobe atrophy on MRI in Parkinson disease with dementia: a comparison with Alzheimer disease and dementia with Lewy bodies. Neurology 2005;64:861-865.

20. Barber R, McKeith IG, Ballard C, Gholkar A, O’Brien JT. A comparison of medial and lateral temporal lobe atrophy in dementia with Lewy bodies and Alzheimer's disease: magnetic resonance imaging volumetric study. Dement Geriatr Cogn Disord 2001;12:198-205.

21. Almeida OP, Burton EJ, McKeith I, Gholkar A, Burn D, O’Brien JT. MRI study of caudate nucleus volume in Parkinson's disease with and without dementia with Lewy bodies and Alzheimer's disease. Dement Geriatr Cogn Disord 2003;16:57-63.

22. Cousins DA, Burton EJ, Burn D, Gholkar A, McKeith IG, O’Brien JT. Atrophy of the putamen in dementia with Lewy bodies but not Alzheimer's disease: an MRI study. Neurology 2003;61:1191-1195.

23. Hanyu H, Shimizu S, Tanaka Y, Hirao K, Iwamoto T, Abe K. MR features of the substantia innominata and therapeutic implications in dementias. Neurobiol Aging 2007;28:548-554.

24. Ashburner J, Friston KJ. Voxel-based morphometry--the methods. Neuroimage 2000;11(6 pt 1):805-821.

25. Ashburner J, Friston KJ. Why voxel-based morphometry should be used. Neuroimage 2001;14:1238-1243. 
26. Burton EJ, Karas G, Paling SM, Barber R, Williams ED, Ballard CG, et al. Patterns of cerebral atrophy in dementia with Lewy bodies using voxel-based morphometry. Neuroimage 2002;17:618-630.

27. Whitwell JL, Weigand SD, Shiung MM, Boeve BF, Ferman TJ, Smith GE, et al. Focal atrophy in dementia with Lewy bodies on MRI: a distinct pattern from Alzheimer's disease. Brain 2007;130(Pt 3):708-719.

28. Brenneis C, Wenning GK, Egger KE, Schocke M, Trieb T, Seppi K, et al. Basal forebrain atrophy is a distinctive pattern in dementia with Lewy bodies. Neuroreport 2004;15:1711-1714.

29. Beyer MK, Larsen JP, Aarsland D. Gray matter atrophy in Parkinson disease with dementia and dementia with Lewy bodies. Neurology 2007;69:747-754.

30. Le Bihan D. Looking into the functional architecture of the brain with diffusion MRI. Nat Rev Neurosci 2003;4:469-480.

31. Conturo TE, Lori NF, Cull TS, Akbudak E, Snyder AZ, Shimony JS, et al. Tracking neuronal fiber pathways in the living human brain. Proc Natl Acad Sci U S A 1999;96:10422-10427.

32. Jones DK, Horsfield MA, Simmons A. Optimal strategies for measuring diffusion in anisotropic systems by magnetic resonance imaging. Magn Reson Med 1999;42:515-525.

33. Mori S, Crain BJ, Chacko VP, van Zijl PC. Three-dimensional tracking of axonal projections in the brain by magnetic resonance imaging. Ann Neurol 1999;45:265-269.

34. Bozzali M, Cherubini A. Diffusion tensor MRI to investigate dementias: a brief review. Magn Reson Imaging 2007;25:969-977.

35. Burn DJ, O'Brien JT. Use of functional imaging in Parkinsonism and dementia. Mov Disord 2003;18 Suppl 6:S88-S95.

36. Firbank MJ, Blamire AM, Krishnan MS, Teodorczuk A, English P, Gholkar A, et al. Diffusion tensor imaging in dementia with Lewy bodies and Alzheimer's disease. Psychiatry Res 2007;155:135-145.

37. Firbank MJ, Blamire AM, Krishnan MS, Teodorczuk A, English P, Gholkar A, et al. Atrophy is associated with posterior cingulate white matter disruption in dementia with Lewy bodies and Alzheimer's disease. Neuroimage 2007;36:1-7.

38. Bozzali M, Falini A, Cercignani M, Baglio F, Farina E, Alberoni M, et al. Brain tissue damage in dementia with Lewy bodies: an in vivo diffusion tensor MRI study. Brain 2005;128(Pt 7):1595-1604.

39. Bozzali M, Falini A, Franceschi M, Cercignani M, Zuffi M, Scotti G et al. White matter damage in Alzheimer's disease assessed in vivo using diffusion tensor magnetic resonance imaging. J Neurol Neurosurg Psychiatry 2002;72:742-746.

40. Ota M, Sato N, Ogawa M, Murata M, Kuno S, Kida J, et al. Degeneration of dementia with Lewy bodies measured by diffusion tensor imaging. NMR Biomed 2009;22:280-284.

41. Kobayashi S, Tateno M, Utsumi K, Takahashi A, Saitoh M, Morii H, et al. Quantitative analysis of brain perfusion SPECT in Alzheimer's disease using a fully automated regional cerebral blood flow quantification software, 3DSRT. J Neurol Sci 2008;264:27-33.

42. Minoshima S, Foster NL, Kuhl DE. Posterior cingulate cortex in Alzheimer's disease. Lancet 1994;344:895.

43. Kemp PM, Holmes C, Hoffmann SM, Bolt L, Holmes R, Rowden J, et al. Alzheimer's disease: differences in technetium-99m HMPAO SPECT scan findings between early onset and late onset dementia. J Neurol Neurosurg Psychiatry 2003;74:715-719.

44. Rodriguez G, Vitali P, Calvini P, Bordoni C, Girtler N, Taddei G, et al Hippocampal perfusion in mild Alzheimer's disease. Psychiatry Res 2000;100:65-74

45. Friston K. Analyzing brain images: principles and overview. In: Frackowiak R, Friston K, Frith C, Dolan R, Mazziotta J, editors. Human Brain Function. San Diego: Academic Press, 1997. p.25-41.

46. Martin AJ, Friston KJ, Colebatch JG, Frackowiak RS. Decreases in regional cerebral blood flow with normal aging. J Cereb Blood Flow Metab 1991;11:684-689.

47. Minoshima S, Koeppe RA, Frey KA, Kuhl DE. Anatomic standardization: linear scaling and nonlinear warping of functional brain im- ages. J Nucl Med 1994;35:1528-1537.

48. Kasama S, Tachibana H, Kawabata K, Yoshikawa H. Cerebral blood flow in Parkinson's disease, dementia with Lewy bodies, and Alzheimer's disease according to three-dimensional stereotactic surface projection imaging. Dement Geriatr Cogn Disord 2005;19:266-275.

49. Mito Y, Yoshida K, Yabe I, Makino K, Tashiro K, Kikuchi S, et al. Brain SPECT analysis by 3D-SSP and phenotype of Parkinson's disease. J Neurol Sci 2006;241:67-72.

50. Mito Y, Yoshida K, Yabe I, Makino K, Hirotani M, Tashiro K, et al. Brain 3D-SSP SPECT analysis in dementia with Lewy bodies, Parkinson's disease with and without dementia, and Alzheimer's disease. Clin Neurol Neurosurg 2005;107:396-403.

51. Kaneko K, Kuwabara Y, Sasaki M, Ogomori K, Ichimiya A, Koga H, et al. Posterior cingulate hypoperfusion in Alzheimer's disease, senile dementia of Alzheimer type, and other dementias evaluated by threedimensional stereotactic surface projections using Tc-99m HMPAO SPECT. Clin Nucl Med 2004;29:362-366.

52. Waragai M, Yamada T, Matsuda H. Evaluation of brain perfusion SPECT using an easy Z-score imaging system (eZIS) as an adjunct to early-diagnosis of neurodegenerative diseases. J Neurol Sci 2007;260: 57-64.

53. Matsuda H, Mizumura S, Nagao T, Ota T, Iizuka T, Nemoto K, et al. Automated discrimination between very early Alzheimer disease and controls using an easy Z-score imaging system for multicenter brain perfusion single-photon emission tomography. AJNR Am J Neuroradiol 2007;28:731-736.

54. Matsuda H, Mizumura S, Nagao T, Ota T, Iizuka T, Nemoto K, et al. An easy Z-score imaging system for discrimination between very early Alzheimer's disease and controls using brain perfusion SPECT in a multicentre study. Nucl Med Commun 2007;28:199-205.

55. Kobayashi S, Tateno M, Utsumi K, Takahashi A, Morii H, Saito T. Two-layer appearance on brain perfusion SPECT in idiopathic normal pressure hydrocephalus: a qualitative analysis by using easy Z-score imaging system, eZIS. Dement Geriatr Cogn Disord 2009;28:330-337.

56. Takeuchi R, Yonekura Y, Takeda SK, Fujita K, Konishi J. Fully automated quantification of regional cerebral blood flow with three-dimensional stereotaxic region of interest template: validation using magnetic resonance imaging--technical note. Neurol Med Chir (Tokyo) 2003;43:153-162.

57. Takeuchi R, Matsuda H, Yoshioka K, Yonekura Y. Cerebral blood flow SPET in transient global amnesia with automated ROI analysis by 3DSRT. Eur J Nucl Med Mol Imaging 2004;31:578-589.

58. Tateno M, Honma T, Kobayashi S, Utsumi K, Fujii K, Morii H. Decreased blood perfusion in right thalamus after transient global amnesia demonstrated by an automated program, 3DSRT. Psychiatry Clin Neurosci 2008;62:244.

59. Tateno M, Kobayashi S, Utsumi K, Morii H, Fujii K. Quantitative analysis of the effects of donepezil on regional cerebral blood flow in Alzheimer's disease by using an automated program, 3DSRT. Neuroradiology 2008;50:723-727.

60. Defebvre LJ, Leduc V, Duhamel A, Lecouffe P, Pasquier F, LamyLhullier C, et al. Technetium HMPAO SPECT study in dementia with Lewy bodies, Alzheimer's disease and idiopathic Parkinson's disease. J Nucl Med 1999;40:956-962.

61. Talbot PR, Lloyd JJ, Snowden JS, Neary D, Testa HJ. A clinical role for 99mTc-HMPAO SPECT in the investigation of dementia? J Neurol Neurosurg Psychiatry 1998;64:306-313.

62. Donnemiller E, Heilmann J, Wenning GK, Berger W, Decristoforo C, Moncayo R, et al. Brain perfusion scintigraphy with 99mTc-HMPAO or $99 \mathrm{mTc}-\mathrm{ECD}$ and 123I-beta-CIT single-photon emission tomography in dementia of the Alzheimer-type and diffuse Lewy body disease. Eur J Nucl Med 1997;24:320-325.

63. Ishii K, Yamaji S, Kitagaki H, Imamura T, Hirono N, Mori E. Regional cerebral blood flow difference between dementia with Lewy bodies and AD. Neurology 1999;53:413-416. 
64. Lobotesis K, Fenwick JD, Phipps A, Ryman A, Swann A, Ballard C, et al. Occipital hypoperfusion on SPECT in dementia with Lewy bodies but not AD. Neurology 2001;56:643-649.

65. Varma AR, Talbot PR, Snowden JS, Lloyd JJ, Testa HJ, Neary D. A 99mTc-HMPAO single-photon emission computed tomography study of Lewy body disease. J Neurol 1997;244:349-359.

66. Colloby SJ, Fenwick JD, Williams ED, Paling SM, Lobotesis K, Ballard C, et al. A comparison of (99m)Tc-HMPAO SPET changes in dementia with Lewy bodies and Alzheimer's disease using statistical parametric mapping. Eur J Nucl Med Mol Imaging 2002;29:615-622.

67. Pasquier J, Michel BF, Brenot-Rossi I, Hassan-Sebbag N, Sauvan R, Gastaut JL. Value of (99m)Tc-ECD SPET for the diagnosis of dementia with Lewy bodies. Eur J Nucl Med Mol Imaging 2002;29:13421348 .

68. Tateno M, Kobayashi S, Shirasaka T, Furukawa Y, Fujii K, Morii H, et al. Comparison of the usefulness of brain perfusion SPECT and MIBG myocardial scintigraphy for the diagnosis of dementia with Lewy bodies. Dement Geriatr Cogn Disord 2008;26:453-457.

69. Tateno M, Utsumi K, Kobayashi S, Takahashi A, Saitoh M, Morii H, et al. Usefulness of a blood flow analyzing program 3DSRT to detect occipital hypoperfusion in dementia with Lewy bodies. Prog Neuropsychopharmacol Biol Psychiatry 2008;32:1206-1209.

70. Herholz K. PET studies in dementia. Ann Nucl Med 2003;17:79-89.

71. Albin RL, Minoshima S, D’Amato CJ, Frey KA, Kuhl DA, Sima AA. Fluoro-deoxyglucose positron emission tomography in diffuse Lewy body disease. Neurology 1996;47:462-466.

72. Imamura T, Ishii K, Sasaki M, Kitagaki H, Yamaji S, Hirono N, et al. Regional cerebral glucose metabolism in dementia with Lewy bodies and Alzheimer's disease: a comparative study using positron emission tomography. Neurosci Lett 1997;235:49-52.

73. Ishii K, Imamura T, Sasaki M, Yamaji S, Sakamoto S, Kitagaki H, et al. Regional cerebral glucose metabolism in dementia with Lewy bodies and Alzheimer's disease. Neurology 1998;51:125-130.

74. Imamura T, Ishii K, Hirono N, Hashimoto M, Tanimukai S, Kazuai H, et al. Visual hallucinations and regional cerebral metabolism in dementia with Lewy bodies (DLB). Neuroreport 1999;10:1903-1907.

75. Higuchi M, Tashiro M, Arai H, Okamura N, Hara S, Higuchi S, et al. Glucose hypometabolism and neuropathological correlates in brains of dementia with Lewy bodies. Exp Neurol 2000;162:247-256.

76. Minoshima S, Foster NL, Sima AA, Frey KA, Albin RL, Kuhl DE. Alzheimer's disease versus dementia with Lewy bodies: cerebral metabolic distinction with autopsy confirmation. Ann Neurol 2001;50: 358-365.

77. Ransmayr G, Seppi K, Donnemiller E, Luginger E, Marksteiner J, Riccabona $\mathrm{G}$, et al. Striatal dopamine transporter function in dementia with Lewy bodies and Parkinson's disease. Eur J Nucl Med 2001;28: 1523-1528.

78. O’Brien JT, Colloby S, Fenwick J, Williams ED, Firbank M, Burn D, et al. Dopamine transporter loss visualized with FP-CIT SPECT in the differential diagnosis of dementia with Lewy bodies. Arch Neurol 2004;61:919-925.

79. McKeith I, O’Brien J, Walker Z, Tatsch K, Booij J, Darcourt J, et al. Sensitivity and specificity of dopamine transporter imaging with $123 \mathrm{I}$ FP-CIT SPECT in dementia with Lewy bodies: a phase III, multicentre study. Lancet Neurol 2007;6:305-313.

80. Nestor PJ. Dopamine transporter brain imaging--can it improve the differential diagnosis of dementia with Lewy bodies? Nat Clin Pract Neurol 2007;3:602-603.

81. Kline RC, Swanson DP, Wieland DM, Thrall JH, Gross MD, Pitt B, et al. Myocardial imaging in man with I-123 meta-iodobenzylguanidine.
J Nucl Med 1981;22:129-132.

82. Wieland DM, Brown LE, Rogers WL, Worthington KC, Wu JL, Clinthorne $\mathrm{NH}$, et al. Myocardial imaging with a radioiodinated norepinephrine storage analog. J Nucl Med 1981;22:22-31.

83. Tobes MC, Jaques S Jr, Wieland DM, Sisson JC. Effect of uptake-one inhibitors on the uptake of norepinephrine and metaiodobenzylguanidine. J Nucl Med 1985;26:897-907.

84. Minardo JD, Tuli MM, Mock BH, Weiner RE, Pride HP, Wellman HN, et al. Scintigraphic and electrophysiological evidence of canine myocardial sympathetic denervation and reinnervation produced by myocardial infarction or phenol application. Circulation 1988;78:1008-1019.

85. Stanton MS, Tuli MM, Radtke NL, Heger JJ, Miles WM, Mock BH, et al. Regional sympathetic denervation after myocardial infarction in humans detected noninvasively using I-123-metaiodobenzylguanidine. J Am Coll Cardiol 1989;14:1519-1526.

86. Merlet P, Valette H, Dubois-Randé JL, Moyse D, Duboc D, Dove P, et al. Prognostic value of cardiac metaiodobenzylguanidine imaging in patients with heart failure. J Nucl Med 1992;33:471-477.

87. Yoshita M. Differentiation of idiopathic Parkinson's disease from striatonigral degeneration and progressive supranuclear palsy using iodine-123 meta-iodobenzylguanidine myocardial scintigraphy. J Neurol Sci 1998;155:60-67.

88. Orimo S, Ozawa E, Nakade S, Sugimoto T, Mizusawa H. (123)I-metaiodobenzylguanidine myocardial scintigraphy in Parkinson's disease. J Neurol Neurosurg Psychiatry 1999;67:189-194.

89. Braune S, Reinhardt M, Schnitzer R, Riedel A, Lücking CH. Cardiac uptake of [123I]MIBG separates Parkinson's disease from multiple system atrophy. Neurology 1999;53:1020-1025.

90. Yoshita M, Taki J, Yamada M. A clinical role for [(123)I]MIBG myocardial scintigraphy in the distinction between dementia of the Alzheimer's-type and dementia with Lewy bodies. J Neurol Neurosurg Psychiatry 2001;71:583-588.

91. Taki J, Yoshita M, Yamada M, Tonami N. Significance of 123I-MIBG scintigraphy as a pathophysiological indicator in the assessment of Parkinson's disease and related disorders: it can be a specific marker for Lewy body disease. Ann Nucl Med 2004;18:453-461.

92. Suzuki M, Kurita A, Hashimoto M, Fukumitsu N, Abo M, Ito Y, et al. Impaired myocardial 123I-metaiodobenzylguanidine uptake in Lewy body disease: comparison between dementia with Lewy bodies and Parkinson's disease. J Neurol Sci 2006;240:15-19.

93. Kobayashi S, Tateno M, Morii H, Utsumi K, Saito T. Decreased cardiac MIBG uptake, its correlation with clinical symptoms in dementia with Lewy bodies. Psychiat Res Neuroim 2009;174:76-80.

94. Hanyu H, Shimizu S, Hirao K, Sakurai H, Iwamoto T, Chikamori T, et al. The role of 123I-metaiodobenzylguanidine myocardial scintigraphy in the diagnosis of Lewy body disease in patients with dementia in a memory clinic. Dement Geriatr Cogn Disord 2006;22:379-384.

95. Yoshita M, Taki J, Yokoyama K, Noguchi-Shinohara M, Matsumoto Y, Nakajima K, et al. Value of 123I-MIBG radioactivity in the differential diagnosis of DLB from AD. Neurology 2006;66:1850-1854.

96. Hanyu H, Shimizu S, Hirao K, Kanetaka H, Iwamoto T, Chikamori T, et al. Comparative value of brain perfusion SPECT and [(123)I]MIBG myocardial scintigraphy in distinguishing between dementia with Lewy bodies and Alzheimer's disease. Eur J Nucl Med Mol Imaging 2006;33:248-253.

97. Estorch M, Camacho V, Paredes P, Rivera E, Rodriguez-Revuelto A, Flotats A, et al. Cardiac (123)I-metaiodobenzylguanidine imaging allows early identification of dementia with Lewy bodies during life. Eur J Nucl Med Mol Imaging 2008;35:1636-1641. 